Trauma Berufskrankh 2016 · [Suppl 4]: 18:S289 DOI 10.1007/s10039-016-0155-4

Online publiziert: 26. April 2016

(c) Springer-Verlag Berlin Heidelberg 2016

CrossMark

„Baden-Baden ist keine Stadt, sondern ein Mikrokosmos. Du denkst zuerst, er ist leicht zu fassen. Doch wenn du in ihn eintauchst, erliegst du seiner Faszination" (Merian, August 2010). Die Faszination, die das Reisemagazin „Merian“ hier beschreibt, muss auch für den Landesverband Südwest der Deutschen Gesetzlichen Unfallversicherung (DGUV) gelten. Denn schon zum 36. Mal sind wir mit der Unfallmedizinischen Tagung hier zu Gast.

Wenn ich mich umschaue, sehe ich viele bekannte und erwartungsvolle Gesichter. Ich verspreche Ihnen: Unsere 36. Unfallmedizinische Tagung wird Sie mit Sicherheit nicht enttäuschen - dafür haben schon unsere beiden wissenschaftlichen Leiter gesorgt. Sie haben federführend ein Programm zusammengestellt, das sowohl interessante Verwaltungsthemen enthält als auch aktuelle medizinische Fragestellungen. Mein besonderer Gruß gilt deshalb Herrn Professor Grützner, BG Klinik Ludwigshafen, und Herrn Professor Stöckle, BG Klinik Tübingen.

Hier im Bénazet-Saal, der ,guten Stube" Baden-Badens begrüße ich weiter:

Frau Dr. Hoeller und Herrn Oberscheven - beide repräsentieren die $\mathrm{Ab}$ teilung Versicherung und Leistungen in der DGUV; außerdem meine Kollegin Frau Kudzielka, sie ist die Landesdirektorin im Landesverband Nordwest der DGUV.

Ein besonderer Gruß gilt auch den Mitgliedern des Beirats unseres Landesverbandes, an der Spitze der Vorsitzende Herr Bühler.

Vom Krankenhausträger der BG Kliniken Tübingen und Ludwigshafen darf ich einige Vorstandsmitglieder, leitende Mitarbeiter und den Geschäftsführer, Herrn Ritter, herzlich begrüßen.

\title{
T. Köhler
}

Deutsche Gesetzliche Unfallversicherung (DGUV), Landesverband Südwest, Heidelberg, Deutschland

\section{Begrüßung}

Und schließlich grüße ich Sie alle: die Ärztinnen und Ärzte, Therapeutinnen und Therapeuten der ambulanten und stationären Einrichtungen und alle Kolleginnen und Kollegen aus den Berufsgenossenschaften und den Unfallkassen.

Was uns im Landesverband Südwest besonders freut, ist die Tatsache, dass sich $\mathrm{zu}$ einer Unfallmedizinischen Tagung unseres Verbandes noch nie so viele Teilnehmerinnen und Teilnehmer angemeldet haben wie in diesem Jahr. Insgesamt liegen uns für heute 740 und für morgen 690 Anmeldungen vor.

Wir werden heute und morgen eine Fülle von Informationen und von neuen Erkenntnissen bekommen. Aber denken Sie bitte auch daran: Unsere Tagung soll auch den partnerschaftlichen Dialog zwischen allen Beteiligten im Heilverfahren der gesetzlichen Unfallversicherung fördern. Wir sehen es als positiv und hilfreich an, wenn sowohl die ärztliche Seite als auch die Verwaltungsseite erkennen, mit welchen Fragestellungen sich die andere Seite gerade beschäftigt. Nutzen Sie deshalb diese Tagung auch als eine Möglichkeit, miteinander ins Gespräch zu kommen.

Bei unserer letzten Unfallmedizinischen Tagung haben Sie uns auf den Bewertungsbögen wieder Hinweise gegeben, wie wir die Tagung noch interessanter und attraktiver gestalten können. Eine zentrale Anregung haben wir aufgegriffen, nämlich den Wunsch, noch mehr medizinische Themen aufzunehmen, die für die Mediziner in der Niederlassung relevant sind.

Erstmals wird es auf dieser Tagung - neben den 6 Hauptthemen - einen Vortrag der ZNS - Hannelore-KohlStiftung geben. Dabei wird das Präventionsprojekt „Schütz deinen Kopf! Gehirnerschütterungen im Sport“ vor- gestellt. Als Beiratsmitglied der Stiftung freue ich mich hierüber ganz besonders und begrüße Frau Lüngen, die Geschäftsführerin dieser erfolgreichen Stiftung.

Information, Kommunikation, der Blick über den Tellerrand, der partnerschaftliche Dialog aller am Heilverfahren Beteiligten - das sind Inhalt und Ziel der beiden vor uns liegenden Tage. Machen wir das Beste daraus!

\section{Korrespondenzadresse}

\section{T. Köhler}

Deutsche Gesetzliche Unfallversicherung (DGUV), Landesverband Südwest 69004 Heidelberg, Deutschland thomas.koehler@bgrci.de

Interessenkonflikt. T. Köhler gibt an, dass kein Interessenkonflikt besteht.

The supplement containing this article is not sponsored by industry. 